\title{
Prenatal diagnosed caudal regression syndrome
}

\author{
Osman Temizkan ${ }^{1 *}$, Faruk Abike $^{2 *}$ Habibe Ayvacı $^{3}$, Ali Yilmaz ${ }^{4}$, Ersan Demirag $^{3}$, Illhan Sanverdi $^{3}$ \\ ${ }^{1}$ Department of Obstetrics and Gynecology, Sisli Etfal Education and Research Hospital, Istanbul, Turkey \\ ${ }^{2}$ Department of Obstetrics and Gynecology, Florence Nightingale Kadıkoy Hospital, Istanbul, Turkey \\ ${ }^{3}$ Department of Obstetrics and Gynecology, Zeynep Kamil Hospital, Istanbul, Turkey \\ ${ }^{4}$ Department of Obstetrics and Gynecology, Haydarpasa GATA Hospital, Istanbul, Turkey \\ Email: ${ }^{*}$ mdtemizkan@yahoo.com, ${ }^{*}$ farukabike@gmail.com
}

Received 17 December 2012; revised 20 January 2013; accepted 30 January 2013

\begin{abstract}
Background: Caudal regression syndrome (CRS) is a rare complex congenital anomaly which is characterized by agenesis of the sacral and lumbar spine. Pelvis, lower extremity, genitourinary, cardiac anomalies and lower extremity neurological and motor development deficits may be accompanied. The exact etiology is unclear but the maternal insulin-dependent diabetes mellitus (hyperglycaemia during embryogenesis seems to act as a teratogen), genetic factors, vascular hypoperfusion may play a role in the etiology. Case: A 41-year-old gravida 4, para 2, live 1, abortus 1 patient with 35 weeks of gestation. The patient had 12-year history of type 1 diabetes mellitus. In ultrasonographic examination we found the length of the bones (all the upper and lower bones) 8 weeks underdeveloped and didn't observe the lumbar, sacral vertebrae and iliac bones. Lower extremities were crossed in frog-like position, there wasn't lower extremity movements. Due to sacral agenesia the head of the femurs were closer. Also pes equinovarum deformity, single umbilical artery, kidneys in contact at the midline were present. All of these findings indicated the diagnosis of caudal regression syndrome. Conclusion: Diabetes is increased fetal anomalities in pregnancy. Diabetic pregnancy should be more evaluated than pregnancies with no risk. Antenatal care should be done more carefully. Especially, prenatal maternal blood glucose levels and HbA1c is most important for prevention of fetal anomalities.
\end{abstract}

Keywords: Caudal Regression Syndrome; Prenatal Diagnosis; Diabetes; Sacral Agenesis

\section{INTRODUCTION}

Caudal regression syndrome (CRS) is a rare complex congenital anomaly which is characterized by agenesis of the sacral and lumbar spine. Pelvis, lower extremity, ge-

\footnotetext{
*Corresponding author.
}

nitourinary, cardiac anomalies and lower extremity neurological and motor development deficits may be accompanied [1-5]. The exact etiology is unclear but the maternal insulin-dependent diabetes mellitus (hyperglycaemia during embryogenesis seems to act as a teratogen), genetic factors, vascular hypoperfusion may play a role in the etiology. Insulin-dependent diabetic pregnancies have an increased risk for CRS [6-10]. With conventional ultrasonography (USG) assessment, it can be diagnosed easily within early weeks. Magnetic resonance imaging (MRI) may be carried out in all patients for diagnosis and determination of other systemic anomalies [11-14]. We have presented and discussed a fetal caudal regression syndrome was diagnosed during pregnancy.

\section{CASE REPORT}

A 41-year-old gravida 4, para 2, live 1, abortus 1 patient with 35 weeks of gestation was referred to our hospital for evaluation of fetal anomalies from another center. The patient had 12-year history of type 1 diabetes mellitus, $\mathrm{Rh}$ isoimmunization (indirect coombs $1 / 32$ pozitive) and high risk triple test (risk was 1 in 15) result. The patient's compliance was not good and pregnancy followup wasn't regular. In her previous pregnancies there was 39 weeks of gestation intrauterine exitus due to Rh isoimmunization and hydrops fetalis four years ago and a first trimester abortus. Her living two children were healthy. The patient didn't use folic acid before and during pregnancy and had no suspected drug use. Biochemical blood examinations showed high fasting blood glucose and HbA1C (8.3\%). US images of the fetal head demonstrated normal intracranial anatomy. In particular, the lateral ventricles and posterior fossa appeared normal and biometric measurements of the head were normal for gestational age. In ultrasonographic examination we found the length of the bones (all the upper and lower bones) 8 weeks underdeveloped and didn't observe the lumbar, sacral vertebrae and iliac bones (Figure 1). Lower extremities were crossed in frog-like position, there wasn't lower extremity movements. Due to sacral agenesia the 
head of the femurs were closer. Also pes equinovarum deformity, single umbilical artery, kidneys in contact at the midline were present (Figure 2). The placentation and amniotic fluid were normal. The ultrasound imaging of other systems were normal. Fetal umbilical and uterin artery doppler was normal. Middle cerebral artery doppler flow was normal. All ultrasonographic findings were confirmed by MRI. MRI examination showed no lumbosacral vertebral column corpuses upper pole of the kidneys, no iliac bones and the anatomic position of the pelvic soft tissues weren't in their normal form (Figure 3). Both kidneys were viewed as in contact to each other at posterior midline. Lower limbs and bone structures were hypoplastic also there was a hypoplasia in muscular structures and fat tissue displaced (Figure 4). All of these findings indicated the diagnosis of caudal regression syndrome. The karyotype assessment was normal. When the pregnancy reached 39 weeks of gestation, a vaginal delivery was implemented. Ultrasonographic and MRI findings were confirmed in postpartum examination (Figure 5).

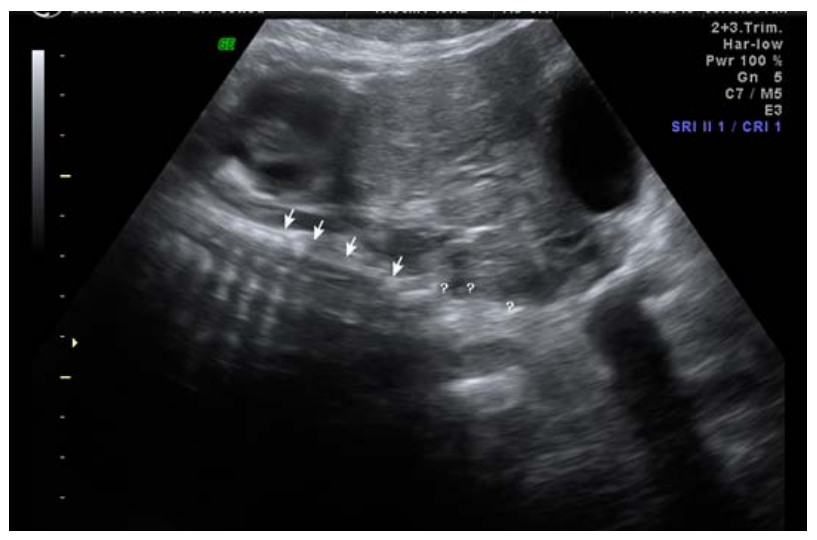

Figure 1. In the ultrasonographic examination there was no lumbar, sacral vertebrae and iliac bones (arrow).

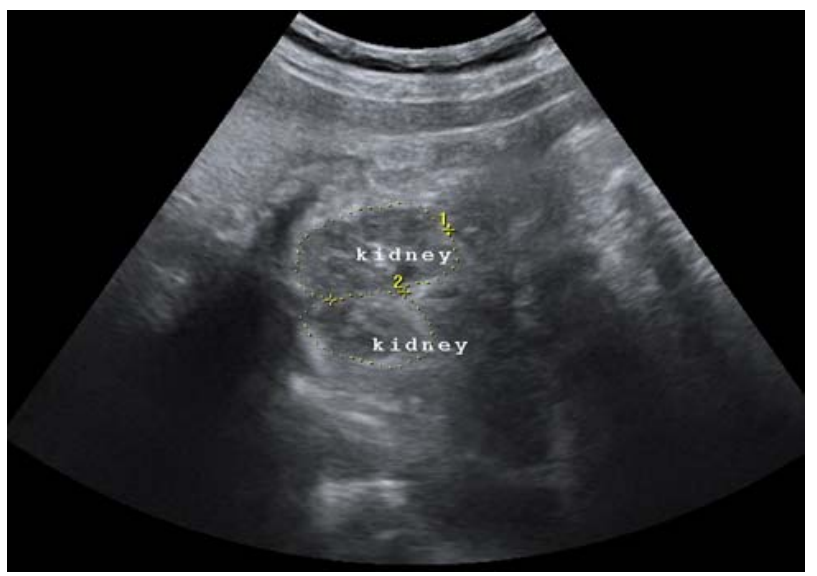

Figure 2. In the ultrasonograhic examination there was combined kidneys at the midline (area 1 and 2 surrounded by the dots).
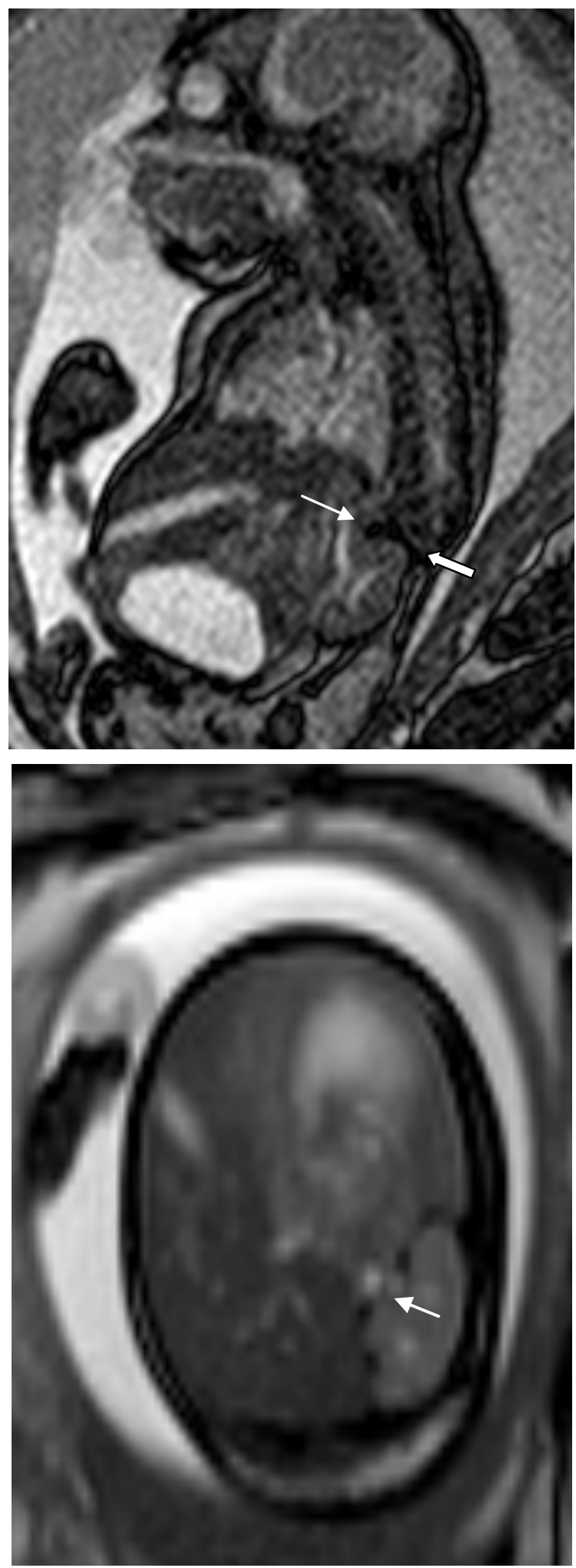

Figure 3. T2 weighted sagittal and oblique sections showed that vertebral column ends at the level of upper pole of the kidneys (small arrow) and there was no sacral vertebrae (big arrow). T2 weighted MRI section showed that there was no vertebral column at the level of kidneys (arrow) so they were contacting to each other. 


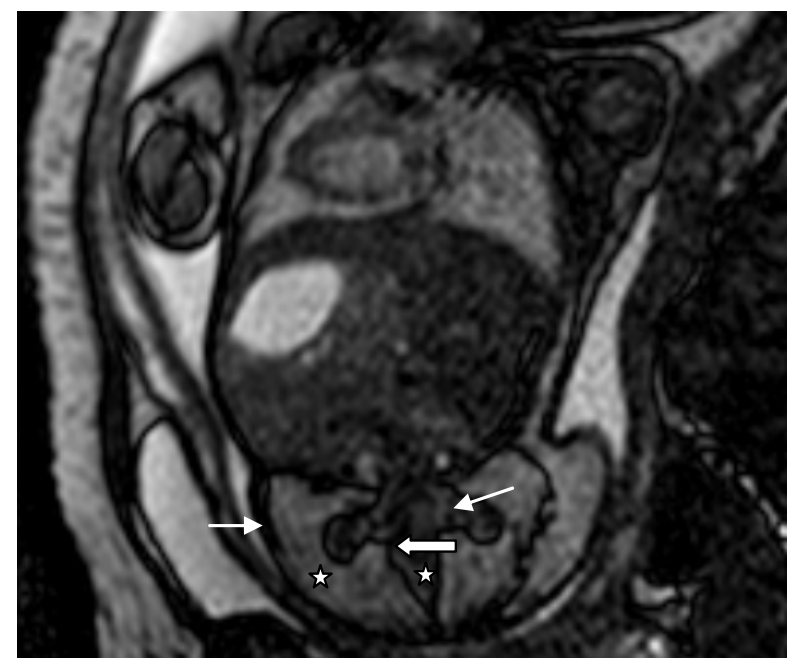

(a)

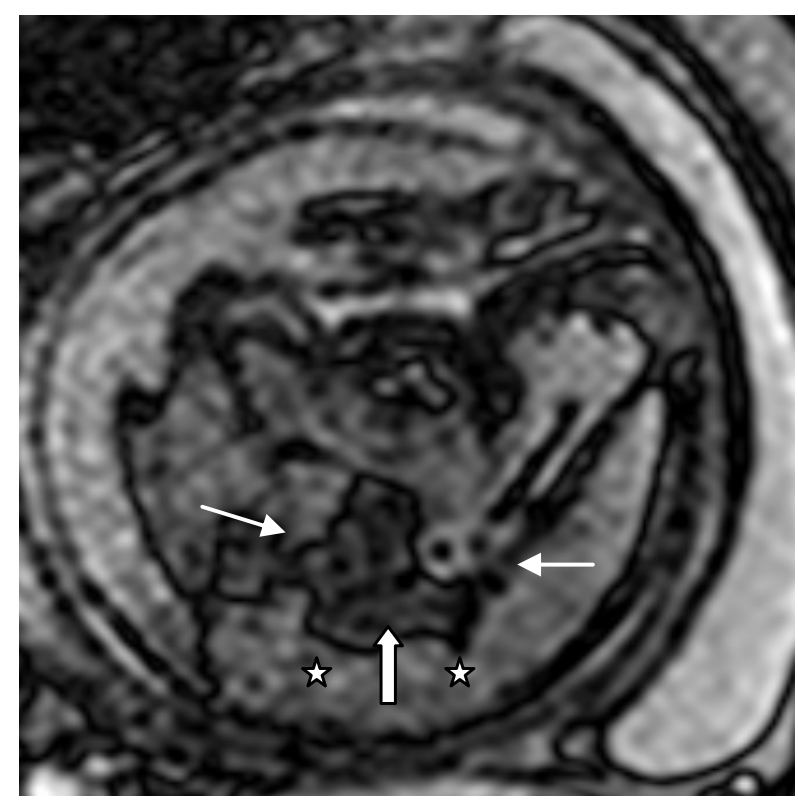

(b)

Figure 4. T2 weighted coronal (a) and axial (b) plans showed that there was hypoplastic fusioned pelvic bones (big arrow) and each femur (small arrow). Also there was a hypoplasia in muscular structures and displaced with hyperintens fat tissue (star sign).

\section{DISCUSSION}

CRS is a rare and which is often sporadic congenital malformation of the lower vertebral column. It is characterized by partial or complete absence of sacrum and lumbar vertebrae. This syndrome is accompanied by severe lower extremity and pelvis deformities, neurological deficits, neural tube defects and genitourinary, gastrointestinal, cardiac anomalies [1,12]. In our case there was sacrum and lumber vertebrae agenesis, short lower extremities with frog-like position and pes equinovarum deformity, a single umbilical artery and kidneys com-

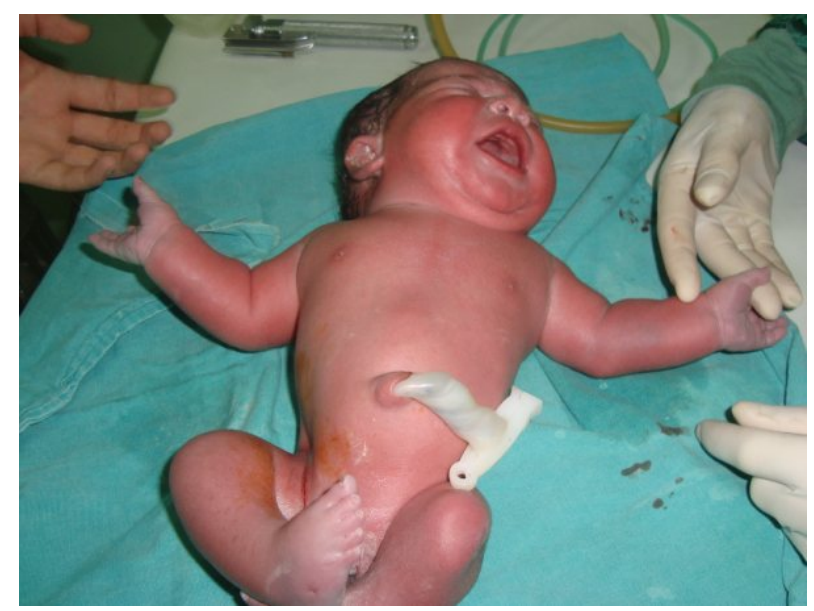

Figure 5. A photo of baby with deformities (short humerus, short low extremities, pes equina varus, narrow pelvis and frog like low extremities).

bined at posterior midline but as a diffence from the litherature there was upper extremity deformity with short humerus.

The mechanism that leads to CRS is not known exactly but chromosomal abnormalities, teratogenic toxins, vitamin deficiencies, physical trauma ( radiation, excessive heat), vascular hypoperfusion and hyperglycemia may play as etiological factors [4-6,9,10].

The defective development of mesodermal midposterior axis causes the syndrome. Probably, embryonal caudal mesoderm developmental abnormalities occur before 4 weeks of pregnancy [2,3]. The severity of the anomalies depends on the degree of the caudal defect in the early period of pregnancy. In various degree of associated anomalies can occur as follows imperforate anus, the absence of external genitalia, renal agenesis, absence of the internal genitalia outside the gonads, single umbilical artery, absence of bladder, adherence of the lower extremity [12].

The diabetic patients with poor glycemic control, in particular before pregnancy, are the most important risk factor. CRS is 200 - 400 times higher in insulin-dependent diabetic pregnant women. More than $20 \%$ of CRS cases were reported in diabetic patients. CRS incidence is approximately $1 / 60.000$ and 2.7 times more common in male fetus [6]. There is no hereditary transition in this syndrome, and CRS is a specific finding for in diabetic embryopathy [6,11]. This syndrome is not associated with aneuploidy and doesn't require fetal karyotyping. However a defect is identified in HLBX9 gene. The gene is localized in chromosome 7q36. This gen is also expressed in the pancreas. Thus, it is suggested that this is the relationship between diabetic hyperglycemia and the CRS. But subsequent studies didn't indicate this relationship [7]. In another study of the same working group, they showed that retinoic acid (RA)-catabolizing enzyme 
CYP26A1 had an important role in protecting tailbud tissues from inappropriate exposure to RA. CYP26A1null animals exhibited caudal agenesis and spina bifida, imperforate anus, agenesis of the caudal portions of the digestive and urogenital tracts, and malformed lumbosacral skeletal elements [8]. This phenotype closely resembles the most severe form of caudal agenesis in humans. In view of these findings, we can say a potential involvement of the human CYP26A1 gene in the pathogenesis of caudal regression syndrome (CRS) but the relationship between CRS risk and the CYP26A1 genotype requires further study with a larger number of genotyped subjects.

The pregestational diabetes is no doubt teratogenic. In the studies implemented with mice, the hyperglycemia induced by retinoic acid resulted in CRS. This effect was found proportional to the dose of retinoic acid. In particular, if hyperglycemic state develops in the early weeks of pregnancy, the risk is greater [9].

The prenatal diagnosis of CRS is clinically possible on 20 to 22 weeks of the pregnancy by USG [12]. There is a case diagnosed in 14 weeks of gestation by three-dimensional ultrasound in the literature. However, sirenomeli fetuses may be diagnosed earlier transvaginally [15]. First-trimester diagnosis is difficult because of insufficient ossification. But CRL and gestational hair disproportion must be cautionary for the diagnosis [13]. At the beginning of the second trimester a shortening of the extremities may be viewed. In our patient the shortening of extremities was the first diagnostic finding too. The patients among high-risk population should be monitored by USG, especially 3D or 4D USD, in order to establish early diagnosis [15]. In particular, sudden angulation on the spine without severe oligohydramnios in pregnancies with type 1 DM risk, and deformation on the lower limbs are clues for CRS [16].

MRI is useful for definitive diagnosis in suspicious patients. The degree of disability in the development of bone and soft tissue is determined better with MRI. The motor insufficiency in these patients is worse than the sensory failure and the sensory level is relatively preserved [10]. The spinal cord level is easily detected with MRI. Therefore for detecting spinal cord abnormalities and determining the fetal prognosis MRI should be done as a diagnostic method [14,17].

The treatment of these patients should performed in a center where multidisciplinary approach can be done. The primary pathology is irreversible and the treatment is only supportive. Orthopedic deformities, bladder and bowel function require long-term treatment. The prognosis depends on the anomaly and the severity of syndrome. Obstetric management should be planned by pregnancy age and after taking the parents' requests into account.

Diabetes is increased fetal anomalities in pregnancy. Diabetic pregnancy should be more evaluated than preg- nancies with no risk. Antenatal care should be done more carefully. Especially, prenatal maternal blood glucose levels and HbA1c is most important for prevention of fetal anomalities.

\section{REFERENCES}

[1] Reece, E.A. and Hobbins, J.C. (1986) Diabetic embryopathy: Pathogenesis, prenatal diagnosis and prevention. Obstetrical \& Gynecological Survey, 41, 325-335. doi:10.1097/00006254-198606000-00001

[2] Valenzano, M., Paoletti, R., Rossi, A., Farinini, D., Garlaschi, G. and Fulcheri, E. (1999) Sirenomelia pathological features, antenatal ultrasonographic clues, and a review of current embryogenic theories. Human Reproduction Update, 5, 82-86. doi:10.1093/humupd/5.1.82

[3] Welch, J.P. and Aterman, K. (1984) Syndrome of caudal regression: A review, including etiologic considerations and evidence of heterogeneity. Fetal \& Pediatric Pathology, 2, 313-327. doi:10.3109/15513818409022263

[4] Adra, A., Cordero, D., Mejides, A., Yasin, S., Salman, F. and O’Sullivan, M.J. (1994) Caudal regression syndrome: Etiopathogenesis, prenatal diagnosis, and perinatal management. Obstetrical \& Gynecological Survey, 49, 508516. doi:10.1097/00006254-199407000-00028

[5] Goto, M.P. and Goldman, A.S. (1994) Diabetic embryopathy. Current Science, 6, 486-491.

[6] Versiani, B.R., Gilbert-Barness, E., Giuliani, L.R., Peres, L.C. and Pina-Neto, J.M. (2004) Caudal dysplasia sequence: Severe phenotype presenting in offspring of patients with gestational and pregestational diabetes. Clinical Dysmorphology, 13, 1-5. doi:10.1097/00019605-200401000-00001

[7] Merello, E., De Marco, P., Mascelli, S., Raso, A., Calevo, M.G., Torre, M., Cama, A., Lerone, M., Martucciello, G. and Capra, V. (2006) HLXB9 homeobox gene and caudal regression syndrome. Birth Defects Research Part A Clinical and Molecular Teratology, 76, 205-209.

[8] De Marco, P., Merello, E., Mascelli, S., Raso, A., Santamaria, A., Ottaviano, C., Calevo, M.G. and Cama, A. (2006) Mutational screening of the CYP26A1 gene in patients with caudal regression syndrome. Birth Defects Research Part A Clinical and Molecular Teratology, 76, 86-95.

[9] Rojansky, N., Fasouliotis, S.J., Ariel, I. and Nadjari, M. (2002) Extreme caudal agenesis. Possible drug-related etiology? Journal of Reproductive Medicine, 47, 241-245.

[10] Nievelstein, R.A., Valk, J., Smit, L.M. and Vermeij Keers, C. (1994) MR of the caudal regression syndrome: Embryologic implications. AJNR American Journal of Neuroradiology, 15, 1021-1029.

[11] Stroustrup S. A., Grable, I. and Levine, D. (2004) Case 66: Caudal regression syndrome in the fetus of a diabetic mother. Radiology, 230, 229-233. doi:10.1148/radiol.2301020942

[12] Singh, S.K., Singh, R.D. and Sharma, A. (2005) Caudal regression syndrome-Case report and review of literature. Pediatric Surgery International, 21, 578-581. 
doi:10.1007/s00383-005-1451-4

[13] Gonzalez-Quintero, V.H., Lama, T., Dibe, M., Romaguera, R.L., Rodriguez, M.M. and Izquierdo, L.A. (2002) Case report-Sonographic diagnosis of caudal regression in the first trimester of pregnancy. Journal of Ultrasound in Medicine, 21, 1175-1178.

[14] Minhanli, I., Kurugoglu, S., Kantarci, F. and Kanberoglu, K. (2001) Dorsolumbosacral agenesis. Pediatric Radiology, 31, 286-288. doi:10.1007/s002470000411

[15] Bashiri, A., Sheizaf, B., Burstein, E., Landau, D., Her- shkovitz, R. and Mazor, M. (2009) Three dimensional ultrasound diagnosis of caudal regression syndrome at 14 gestational weeks. Archives of Gynecology and Obstetrics, 280, 505-507. doi:10.1007/s00404-009-0943-1

[16] Arthur, C.F. (2010) Sonograhy in obstetrics and gynecology principles and practice, 7th Edition, McGraw Hill, New York.

[17] Duczkowska, A., Bekiesinska, M., et al. (2011) Magnetic resonance imaging in the evaluation of the fetal spinal canal contents. Brain and Devoelopment, 33, 10-20. 